\title{
Characteristics of consecutive bursts of Pi2 pulsations observed at the SMALL array: A new implication
}

\author{
C.-C. Cheng ${ }^{1}$, C. T. Russell ${ }^{2}$, K. Yumoto ${ }^{3}$, Y. F. Gao ${ }^{4}$, and P. J. Chi ${ }^{2}$ \\ ${ }^{1}$ Department of Physics, National Huwei University of Science and Technology, Hu-Wei, Taiwan 63201, ROC \\ ${ }^{2}$ Institute of Geophysics and Planetary Physics, University of California, Los Angeles, CA 90095, USA \\ ${ }^{3}$ Department of Earth and Planetary Sciences, Kyushu University 33, Fukuoka 812-8581, Japan \\ ${ }^{4}$ Institute of Geophysics, China Seismological Bureau, China
}

(Received August 1, 2003; Revised February 12, 2004; Accepted April 19, 2004)

\begin{abstract}
Consecutive bursts of $\mathrm{Pi} 2$ pulsations are examined with magnetic field data obtained by the SMALL array in 1999. With reference to the $H$-component magnetic bays in the high-latitude magnetograms at CPMN, 10 events consisting of two consecutive Pi2 bursts simultaneously observed by the Beijing (BJI, $L=1.46$ ) and Wuhan (WHN, $L=1.20$ ) stations are identified as associated with substorm onsets. Owing to the same waveform seen by the CPMN and IGPP/LANL arrays, they are the global phenomena. Their occurrences are mostly in the 2100-2300 LT (local time) sector in which the dominant frequencies at WHN are higher than the mean frequency, but those at BJI are lower and close to the frequency of the surface wave at the plasmapause. Moreover, the LT dependence of azimuth and polarization of two consecutive Pi2 bursts at BJI and WHN are analyzed and consistent with the ULF waves theory by Itonaga and Yumoto (1998). GOES 8 and GOES 10 confirm the formation of the substorm current wedge after the onsets of two Pi2 bursts. Thus during substorm onsets, Pi2 pulsations at low latitudes may result from hydromagnetic waves driven by an impulsive source in the magnetotail which could commence in the longitude of 2230 LT and later propagate westward and eastward as well. Low-latitude Pi2 waves near the source site may be affected by several factors as they propagate by the stimulation of a surface wave at the plasmapause, by a localized field line oscillation inside the plasmapause, and by the magnetospheric/plasmaspheric cavity (resonance) mode.
\end{abstract}

Key words: Pi2 pulsations, substorm onsets, ULF waves.

\section{Introduction}

For decades, determining the generation and propagation mechanisms of Pi2 pulsations has been an active area of space physics research. Pi2 pulsations consist of impulsive and damped oscillations of geomagnetic fields in a period range of 40 to 150 seconds, and occur predominantly in the nighttime in association with substorm onsets (e.g., Yumoto, 1986; Olson, 1999).

It appears that several mechanisms may generate $\mathrm{Pi} 2 \mathrm{~s}$ and that several factors affect them before they are observed by a remote observer. The first possible generation mechanism is a bouncing Alfvén wave between the auroral ionosphere and the plasma sheet. This may produce high-latitude Pi2 pulsations (Baumjohann and Glassmeier, 1984; Bauer et al., 1995). The substorm current wedge oscillation that is the geomagnetic perturbation induced by the current system in the polar region after substorm onset, has been suggested as a second source mechanism that may be responsible for midand low-latitude Pi2 pulsations (e.g., Lanzerotti and Medford, 1984; Lester et al., 1989 and references therein). Support for this is found in polarization patterns and the orientation of the major axis. By using comparison with ground and satellite observations, Kepko and Kivelson (1999) reported

Copy right (c) The Society of Geomagnetism and Earth, Planetary and Space Sciences (SGEPSS); The Seismological Society of Japan; The Volcanological Society of Japan; The Geodetic Society of Japan; The Japanese Society for Planetary Sciences; TERRAPUB that there is a direct link between mid- and low-latitude Pi2 pulsations and bursty bulk flows (BBFs) in the magnetotail. The braking of earthward BBFs could lead to the formation of the substorm current wedge. Consequently, mid-latitude $\mathrm{Pi} 2 \mathrm{~s}$ were regarded as the transient response of the ionosphere to the substorm current wedge. Furthermore, lowlatitude Pi2s on the flank were found to be in a same period as the oscillating BBF. Thus, Kepko and Kivelson (1999) proposed that BBFs could directly excite mid- and low-latitude $\mathrm{Pi} 2$ pulsations.

A factor that affects the appearance of mid-latitude $\mathrm{Pi} 2 \mathrm{~s}$, is the stimulation of a surface wave at the plasmapause and/or a localized field line oscillation inside the plasmapause. By analyzing the latitudinal profile of peak power of $\mathrm{Pi} 2$ pulsations in the Circum-pan Pacific Magnetometer Network (CPMN), Yumoto and the CPMN Group (2001) found that the peak power of $H$ and $D$ components of $\mathrm{Pi} 2$ pulsations become interchanged from high to low frequency at stations close to plasmapause. In addition, Kosaka et al. (2002) reported that the dominant frequency of mid-latitude $\mathrm{Pi} 2$ is consistent with the resonance frequency of the surface wave at the plasmapause in a plasmaspheric model including the effect of plasmapause bulge. Another factor that affects Pi2 appearance according to some recent studies (e.g., Lin et al., 1991; Li et al., 1998; Lee and Kim, 1999; Nosé, 1999; Cheng et al., 2000; Fujita and Itonaga, 2003 and references therein) is the modification of low-latitude Pi2 from the mag- 
Table 1. Occurrences of two consecutive Pi2 bursts at available stations in 1999.

\begin{tabular}{cccccc}
\hline $\begin{array}{c}\text { Event } \\
\text { number }\end{array}$ & Date & $\begin{array}{c}\text { Start time of } \\
\text { burst } 1(\mathrm{UT})\end{array}$ & $\begin{array}{c}\text { Start time of } \\
\text { burst } 2(\mathrm{UT})\end{array}$ & SMALL array & CPMN array* \\
\hline 1 & Oct. 8 & 1112 & 1138 & BJ, WHN & DAL, WEP \\
2 & Oct. 11 & 1348 & 1400 & BJ, WHN & \\
3 & Oct. 15 & 1316 & 1332 & BJ, WHN & DAL, WEP \\
4 & Oct. 16 & 1425 & 1438 & BJI, WHN & \\
5 & Oct. 17 & 1239 & 1254 & BJI, WHN & DAL, WEP \\
6 & Oct. 23 & 1418 & 1440 & BJI, WHN & DAL, WEP \\
7 & Oct. 25 & 1416 & 1431 & BJI, WHN & DAL, WEP \\
8 & Oct. 28 & 1652 & 1700 & BJI, WHN & DAL, WEP \\
9 & Dec. 5 & 1457 & 1507 & BJI, WHN & \\
10 & Dec. 6 & 1704 & 1726 & BJI, WHN & \\
\hline
\end{tabular}

*South-hemisphere stations with nearly same $L$ as those at SMALL.

Table 2. Locations of available stations at the SMALL, CPMN and IGPP/LANL arrays.

\begin{tabular}{|c|c|c|c|c|c|c|c|}
\hline Station name & Abbr. & $\begin{array}{c}\text { Geographic } \\
\text { lat. }\end{array}$ & $\begin{array}{c}\text { Geographic } \\
\text { long. }\end{array}$ & $\begin{array}{c}\text { Corr. geomag. } \\
\text { lat. }\end{array}$ & $L$ & $\begin{array}{l}\text { LT-UT } \\
\text { (hrs) }\end{array}$ & Array name \\
\hline Beijing & $\mathrm{BJI}$ & 40.0 & 116.2 & 34.2 & 1.46 & +8 & SMALL \\
\hline Wuhan & WHN & 30.5 & 114.6 & 24.2 & 1.20 & +8 & SMALL \\
\hline Kotelnyy & $\mathrm{KTN}$ & 75.94 & 137.71 & 70.25 & 8.76 & +9 & $\mathrm{CPMN}$ \\
\hline Tixie & TIK & 71.59 & 128.78 & 65.99 & 6.04 & +9 & CPMN \\
\hline Chokurdakh & CHD & 70.62 & 147.89 & 64.96 & 5.58 & +9 & CPMN \\
\hline Zyryanka & ZYK & 65.75 & 150.78 & 59.87 & 3.97 & +9 & CPMN \\
\hline Magadan & MGD & 59.97 & 150.86 & 53.75 & 2.86 & +9 & CPMN \\
\hline St. Paratunka & PTK & 52.94 & 158.25 & 46.38 & 2.10 & +9 & CPMN \\
\hline Moshiri & MSR & 44.37 & 142.27 & 37.57 & 1.59 & +9 & CPMN \\
\hline Kagoshima & KAG & 31.48 & 130.72 & 24.70 & 1.21 & +9 & CPMN \\
\hline Weipa & WEP & -12.68 & 141.88 & -21.93 & -1.18 & +9 & CPMN \\
\hline Dalby & DAL & -27.18 & 151.20 & -36.61 & -1.58 & +9 & CPMN \\
\hline Athabasca & ATH & 54.72 & 246.72 & 62.31 & 4.63 & -7 & IGPP/LANL \\
\hline USAFA & AFA & 39.01 & 255.12 & 48.05 & 2.24 & -7 & IGPP/LANL \\
\hline
\end{tabular}

netospheric/plasmaspheric (virtual) cavity resonance. Another factor for low-latitude Pi2 waves is the coupling of cavity (resonance) modes to field line resonances as the eigenfrequency is matched in the inhomogeneous distribution of plasma and field in the inner magnetosphere (e.g., Cheng et al., 1998). The last factor is the propagation of the electric field from the nightside auroral latitude to the equatorial ionosphere in a manner favorable to the generation of dayside events.

By comparing mid- and low-latitude $\mathrm{Pi} 2$ pulsations, Villante et al. (1992) found that there is a strong local time (LT) control of low-latitude $\mathrm{Pi} 2$ polarization pattern, and the orientation of the major axis of low-latitude $\mathrm{Pi} 2$ has a clear variation before and after 2200 LT. For the mid-latitude station, the observed Pi2 polarizations show clear LT control after local midnight that is contrary to the prediction of the substorm current wedge model around 2300 LT. By comparing the AMPTE/CCE and ground-based observations, Takahashi et al. (1995) reported that compressional Pi2 oscillations are seldom seen at $2<L<5$ in the inner magnetosphere outside a region of 6 hours in the midnight sector. Recently, Yumoto and the CPMN Group (2001) showed that high-latitude Pi2 predominantly appear in the midnight sector but mid- and low-latitude Pi2 in the 2100-0200 LT sector. These phenomena indicate that the source location of mid- and low-latitude Pi2 may be not localized around the midnight as those at high latitudes.

Recently, Russell (2000) proposed a two-neutral-point model to explain two distinct onsets (e.g., Mishin et al., 2001 and references therein). In the model that the interplanetary magnetic field (IMF) undergoes a single north to south and back to north sequence of variation. The interplay between near Earth and distant neutral points in the magnetotail creates two substorm onsets, one when reconnection at the near Earth neutral point first begins and one when reconnection reaches the open flux of the tail lobes. Thus, during substorm onsets, there are generally two consecutive $\mathrm{Pi} 2$ bursts or multiple bursts both on the ground and in space. This scenario has been shown to be possible with comparison of the systematic IMF observations and consecutive Pi2 bursts at the SMALL and IGPP/LANL array by Cheng et al. (2002a, b). Especially by using the magnetic field data from the Sino Magnetic Array at Low Latitudes (SMALL) in 1999, Cheng et al. (2002a) examined the correlation between the domi- 


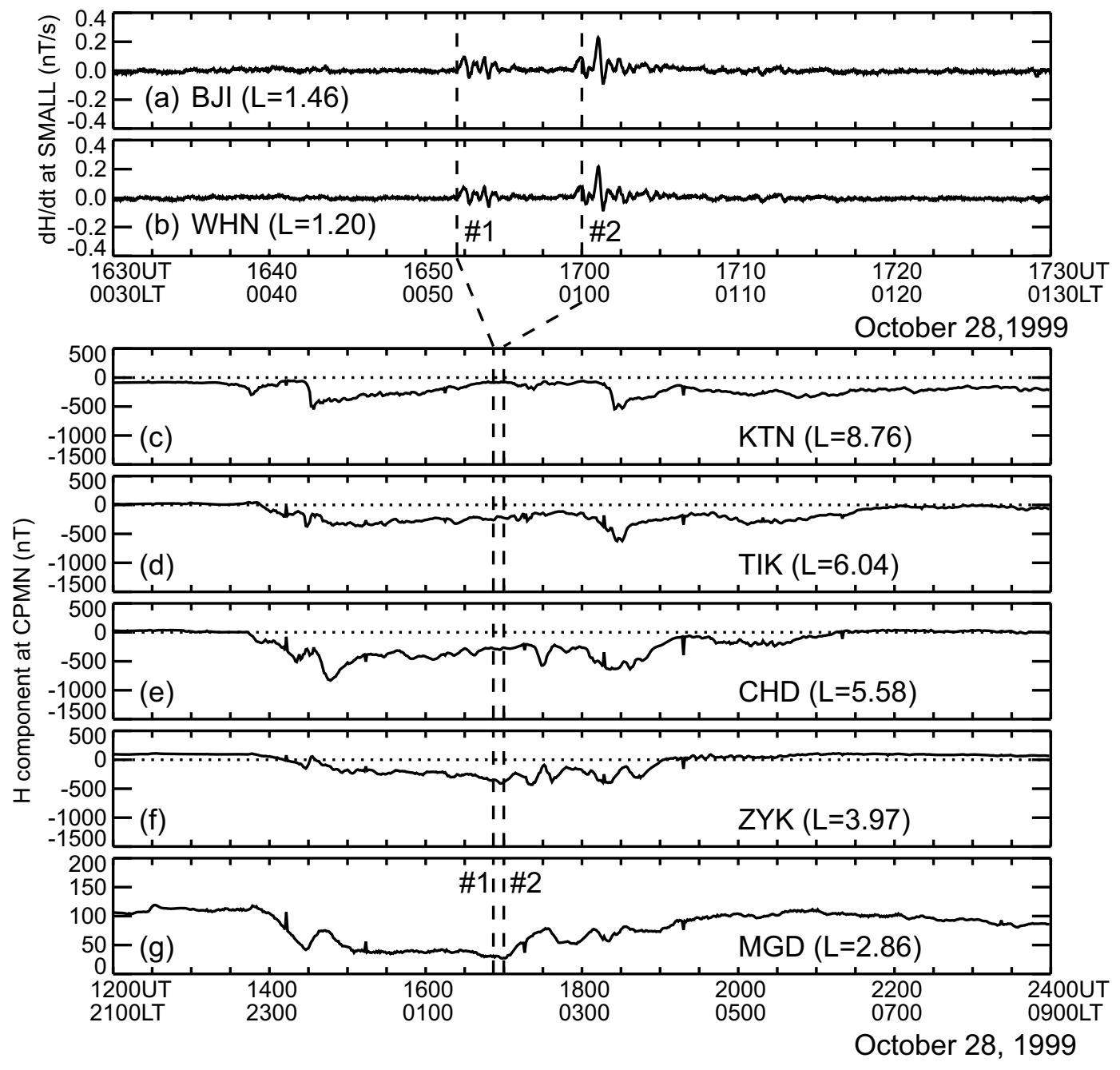

Fig. 1. An example of two consecutive Pi2 bursts simultaneously occurring at BJI $(L=1.46)$ and WHN $(L=1.20)$ as displayed with the time derivative of $H$-component in the context of 1 -minute $H$-component magnetic field data at CPMN on October 28, 1999 . The first Pi2 burst occurred at 1652 UT (0052 LT) and the second at 1700 UT (0100 LT). The dashed line denotes the onset time of two Pi2 bursts. \#1 denotes the first Pi2 burst and \#2 for the second one.

nant frequencies of two consecutive Pi2 bursts. They found that the correlation coefficient for the 'high-latitude' station at SMALL is larger than that at the 'low-latitude' station. This implies that $\mathrm{Pi} 2$ pulsations at low latitudes are more dominated by the cavity wave than those at the higher latitudes. Since most stations of the SMALL array are usually inside the plasmasphere, the generation and propagation mechanisms of $\mathrm{Pi} 2$ pulsations at low latitudes need to be further verified with consideration of their wave polarizations. By reanalyzing the characteristics of two consecutive $\mathrm{Pi} 2$ bursts simultaneously observed at SMALL in the Cheng et al. (2002a) study, our goal is first to clarify their generation mechanism, and second to examine their propagation mechanism. The magnetic field data from the stations at CPMN and IGPP/LANL as well as geostationary satellites will be compared to those at SMALL in this study.

\section{Data Presentation}

In the Cheng et al. (2002a) study, there are 33 events consisting of two consecutive Pi2 bursts at SMALL in association with the $H$-component magnetic bays at the highlatitude stations at CPMN in 1999. Especially ten events are simultaneously observed at the BJI and WHN stations and shown in Table 1 in this study. Other 23 events are not always simultaneously seen by more than two sister stations in the SMALL array and hence not included in this study. The Beijing (BJI) station is located at $34.2^{\circ}$ corrected magnetic latitude and $188.7^{\circ}$ corrected magnetic longitude with $L=1.46$. The Wuhan (WHN) station is located at $24.2^{\circ}$ corrected magnetic latitude and $186.6^{\circ}$ corrected magnetic longitude with $L=1.20$. The geographic locations of the BJI and WHN stations are shown in Table 2. A more detailed description of the SMALL array is provided by Gao et al. (2000). To demonstrate that the two consecutive Pi2 bursts at BJI and WHN are global phenomena, we study one event as seen in the magnetic field data from CPMN, IGPP/LANL, and synchronous orbit satellites. Hence, features of the generation and propagation mechanisms of $\mathrm{Pi} 2$ pulsations can be studied with the simultaneous observations at BJI and WHN.

Figure 1 shows an example of two consecutive Pi2 bursts simultaneously occurring at BJI and WHN (displayed with the time derivative of $H$-component with the simulation of a search coil magnetometer with a band pass up to 0.125 $\mathrm{Hz}$ ) compared with 1-minute $H$-component records of the 


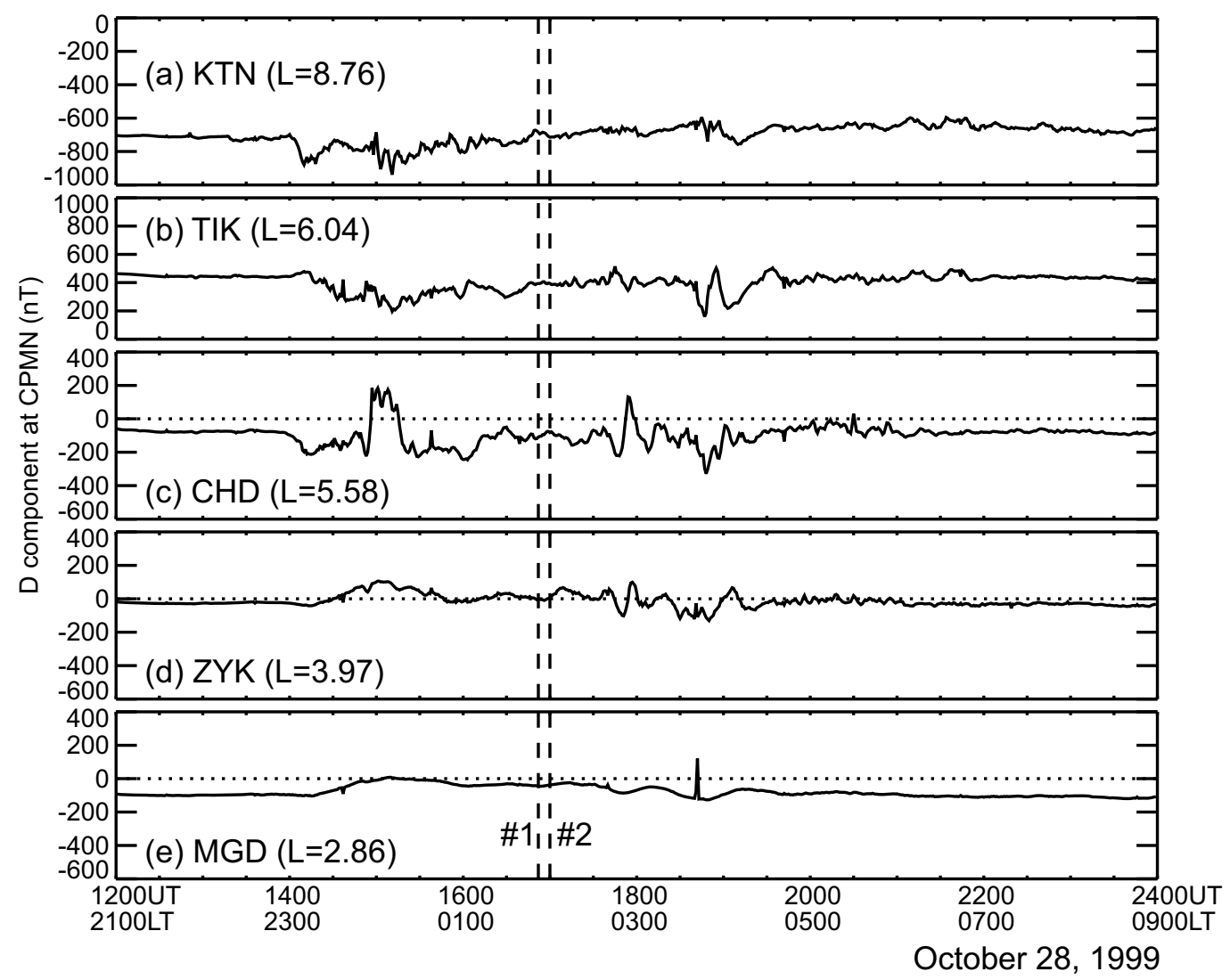

Fig. 2. In the same format as Fig. 1, except for the D-component at CPMN on October 28, 1999.

magnetic field along the CPMN array on October 28, 1999. The onset time of the first Pi2 burst is about 1652 UT (0052 LT) and the second about 1700 UT (0100 LT). Note that the local time at the SMALL array is the universal time (UT) plus 8 hours and that at CPMN plus 9 hours (see Table 2). In Fig. 1, \#1 denotes the first Pi2 burst and \#2 for the second one. The dashed line denotes the onset time of the Pi2 bursts henceforth in this study. The comparison of Figs. 1(a) to 1(b) shows that two consecutive Pi2 bursts in the time derivative of $H$-component at BJI are in phase with those at WHN. This supports the view that low-latitude Pi2 may be the cavity (resonance) modes resulting from fast compressional waves from the magnetotail during substorm onsets.

The substorm onset generally occurs after the IMF turns southward for a period time $\geq 30 \mathrm{~min}$ (e.g., Russell, 2000). According to the IMF observation at Wind which was located at $\sim 28 \mathrm{R}_{\mathrm{E}}$ (Earth's radii) in front of the Earth (not shown in this study), the IMF turned southward at $\sim 1330$ UT on October 28, 1999. And the southward IMF continued to $\sim 1740$ UT with a time of more than 4 hours. It is clear that two consecutive $\mathrm{Pi} 2$ bursts at BJI and WHN are associated with substorm onset during the time of interest. Figures 1(c) to 1 (g) show the occurrence of $H$-component magnetic bays from the high- to low-latitude stations at CPMN. The locations of the available stations at CPMN are shown in Table 2. From Figs. $1(\mathrm{c})$ to $1(\mathrm{~g})$, one may find that the $H$-component of magnetic field at CHD ( $L=5.58$ ) is the first to begin a sharp decrease in two stages at $\sim 1345$ UT and reach the minimum at $\sim 1440$ UT. Subsequently, the $H$-component at
CHD gradually increased but that at ZYK $(L=3.97)$ and MGD $(L=2.86)$ remained decreasing before onsets of two consecutive Pi2 bursts. After onset of the second $\mathrm{Pi} 2$ burst, the $H$ components at ZYK and MGD gradually recovered to be in the undisturbed state. In the same format as Figs. 1(c) to $1(\mathrm{~g})$, Figures 2 and 3 show the $D$ and $Z$ components at CPMN, respectively. In Fig. 2, the $D$-component at TIK decreased and that at CHD increased instead. Moreover, Figure 3 shows that the $Z$-component at CHD increased and that at ZYK decreased. These indicate that during substorm onset the westward traveling surge may be located between CHD and ZYK and the field-aligned current may go upward over CHD. As a result, two consecutive Pi2 bursts at BJI and WHN may be relevant to the substorm current wedge. To verify the view, we will study the local time dependence of the dominant frequency and wave polarizations of two consecutive Pi2 bursts at the BJI and WHN stations in the following section.

\section{Characteristic Analysis}

As in the study of Cheng et al. (2002a), a magnetic field analysis program, BANAL (Russell, 1983), has been used to perform the spectral analysis of low-latitude $\mathrm{Pi} 2$ at the BJI and WHN stations. The advantage to using the fast fourier transform (FFT) in the BANAL is the capability of selecting the time window arbitrarily. The time window for this study is about 4-11 min dependent upon the duration time of each $\mathrm{Pi} 2$ burst. In the frequency domain, there are three spectral estimates averaged to get the final spectra. In addition, BANAL has the capability of demonstrating the 


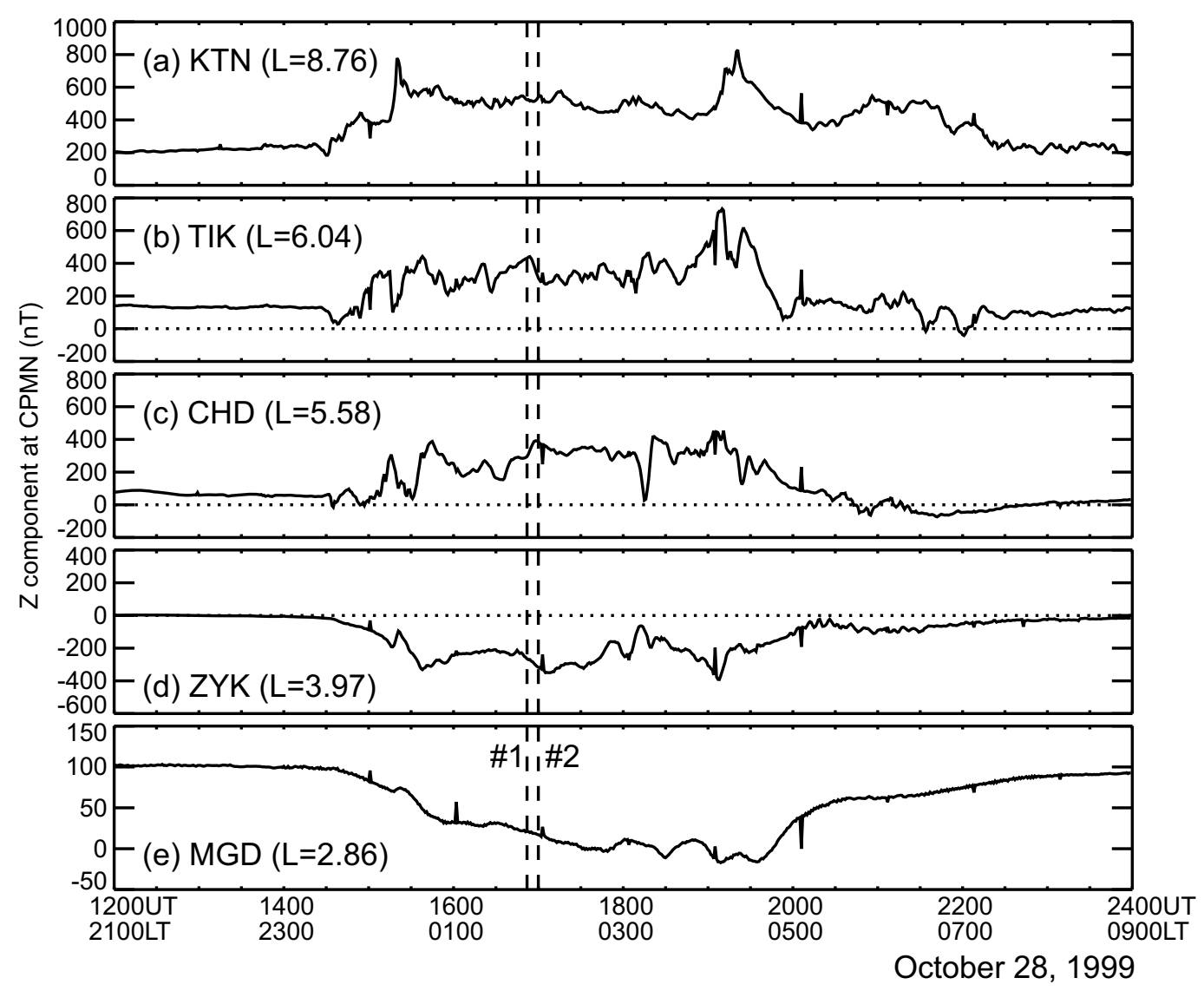

Fig. 3. In the same format as Fig. 1, except for the Z-component at CPMN on October 28, 1999.

$H-D$ hodogram of hydromagnetic waves. It is important to understand the generation and propagation mechanisms of $\mathrm{Pi} 2$ pulsations by using the $H-D$ hodogram in addition to the spectral analysis. Hence, the local time dependence of the dominant frequencies and wave polarizations of two consecutive Pi2 bursts can be studied with BANAL in this study.

\subsection{Dominant frequency}

Using FFT, the dominant frequencies of two consecutive $\mathrm{Pi} 2$ bursts at $\mathrm{BJI}$ and $\mathrm{WHN}$ versus LT and the $K p$ index are shown in Fig. 4. Note that the cross denotes the first Pi2 burst, the triangle for the second burst, the dotted line for the mean dominant frequency $f_{D}$ of $\mathrm{Pi} 2$ burst, and the square for the $K p$ index, respectively. Both panels of Fig. 4 show that $f_{D}$ is about $17.17 \mathrm{mHz}$ for BJI and $17.22 \mathrm{mHz}$ for WHN. One may find from Fig. 4 that most of the two consecutive $\mathrm{Pi} 2$ bursts occur before the midnight and dominantly around 2200 LT. Moreover, there are twelve Pi2 bursts at both stations in the interval 2100-2300 LT in Fig. 4. By close inspection of Fig. 4(a), the dominant frequencies of Pi2 bursts at $\mathrm{BJI}$ are below $f_{D}$ with a margin of 8 to 4 . But the dominant frequencies of $\mathrm{Pi} 2$ bursts at WHN are above $f_{D}$ with small margin of 7 to 5 in Fig. 4(b). Hence, there is a tendency for the dominant frequency at BJI to become below $f_{D}$ and that at WHN to be higher than $f_{D}$ instead. One may argue that the difference of the dominant frequency of $\mathrm{Pi} 2 \mathrm{~s}$ between BJI and WHN may be attributed to the frequency resolution determined by the length of time window. To reduce the uncertainty, we selected a same length of time window for each
Pi2 event at both BJI and WHN. For justification, we also analyzed the same $\mathrm{Pi} 2$ events in the south hemisphere stations at CPMN in a similar way. Unfortunately, only six events occurring simultaneously at the Weipa (WEP, $L=-1.18$ ) and Dalby (DAL, $L=-1.58$ ) stations at CPMN were available for comparison with those at SMALL. The more detail on the location of the WEP and DAL stations can refer to Table 2. In Fig. 4, the open diamond denotes the dominant frequency of Pi2s at WEP and DAL. The pattern of Fig. 4 remains unchanged with inclusion of the data points from the WEP and DAL stations. Hence, the length of time window is not the key factor for the difference of the dominant frequency of Pi2s between BJI and WHN.

In this study, the $K p$ index is about $4-5$ from most events except for one at the dusk sector with $K p=3$. This provides us an opportunity to find out what component plays a dominant role on the LT dependence of Pi2's frequency. In figure 8(d) of the Kosaka et al. (2002) study, the average dominant frequencies of $\mathrm{Pi} 2$ pulsations at the Kakioka (KAK, $L=1.3$ ) station are about $15-17 \mathrm{mHz}$ for the $H$ component in the interval 2100-2300 LT. Moreover, they compared to the estimation of the frequency of the surface wave at the plasmapause as $K p$ varies from 1 to 4 . Since the LT distribution of the estimated frequency of the surface wave for $K p=4$ (shown in Fig. 4 of this study with the dashed line) is inconsistent with that of Pi2's dominant frequency at KAK, Kosaka et al. (2002) suggested that the surface wave at the plasmapause may be not significantly affect low-latitude Pi2. By comparing their figure 8(d) to Fig. 4 


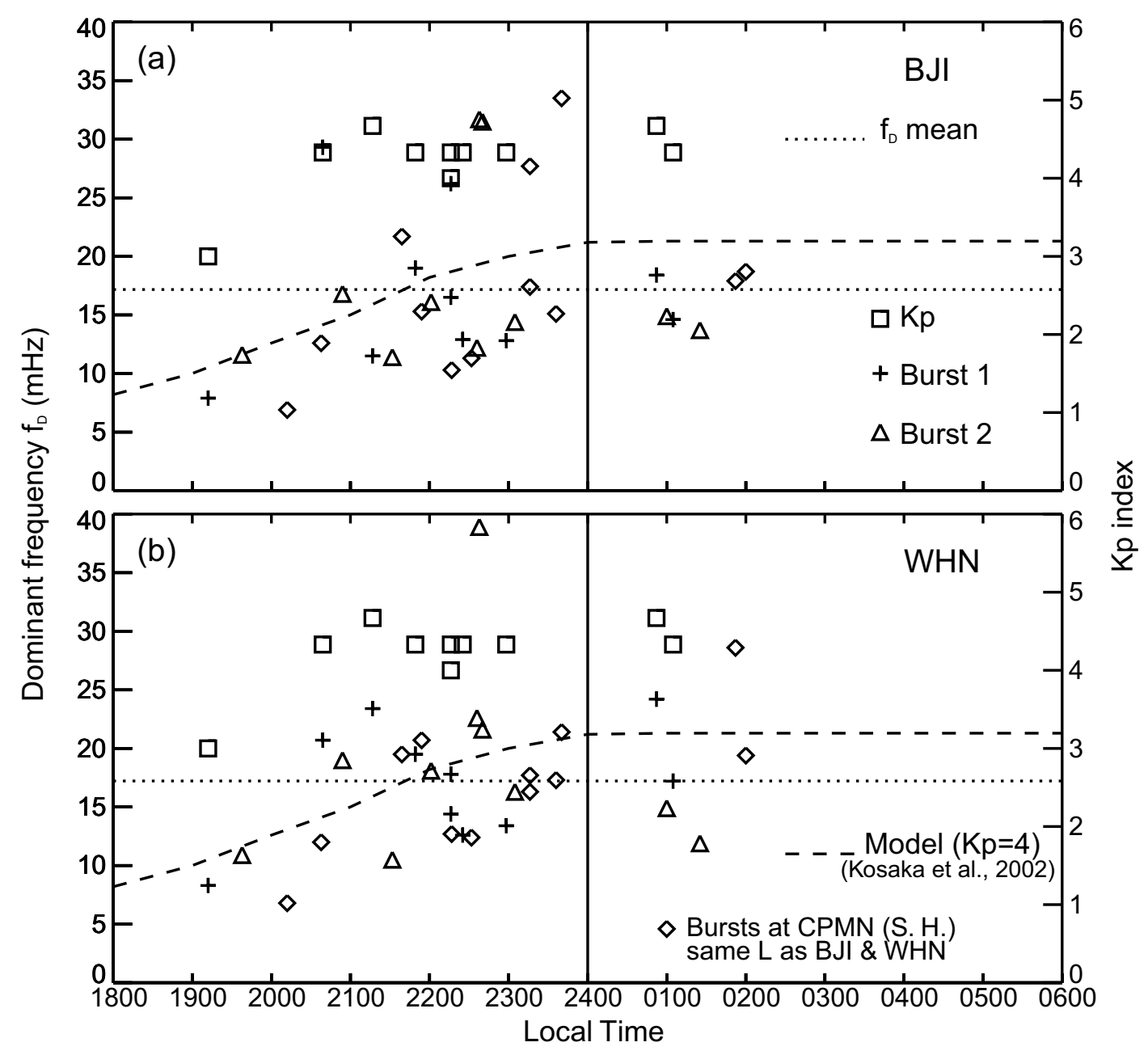

Fig. 4. The dominant frequency of two consecutive Pi2 bursts at BJI $(L=1.46)$ and WHN $(L=1.20)$ and the $K p$ index versus LT, respectively. The cross denotes the first Pi2 burst, the triangle for the second burst, the dotted line for the mean dominant frequency $f_{D}$ of Pi2 burst, the square for the $K p$ index, the open diamond for the bursts in the south hemisphere, and the dashed line for the estimated frequency of the surface wave for $K p=4$ by Kosaka et al. (2002), respectively.

in this study, Pi2's dominant frequency at BJI is similar to that at KAK and in the range of the estimated frequency of the surface wave at the plasmapause in the interval 21002200 LT. The Pi2's dominant frequency at WHN, however, is slightly different from that at KAK and higher than the estimated frequency of the surface wave at the plasmapause. According to Fig. 6 in the Kosaka et al. (2002) study, the plasmapause location moves closer to the Earth as $K p$ becomes higher. During the interval, both BJI and KAK may be closer to the footpoint of the plasmapause for $K p=4$ than WHN. This may be the reason why the LT dependence of Pi2's dominant frequency at KAK resembles BJI and not WHN. On the other hand, Fujita and Itonaga (2003) simulated the behavior of hydromagnetic waves in a model magnetosphere with a longitudinally non-uniform plasmasphere after substorm onset. Their simulation results show that the cavity (resonance) mode in a $\mathrm{Pi} 2$ period could sustain in the plasmapshere. In addition, the plasmaspheric cavity (resonance) mode could have a position-dependent frequency like those observed at KAK and BJI. Unlike the cavity mode, the surface wave is an evanescent fast mode and its amplitude decreases as it propagates isotropically. Until now, the longitudinal extent of localized Pi2s is not well determined. Hence, Fujita and Itonaga (2003) suggested that the surface wave could be a possible generation mechanism for Pi2s localized in a longitudinal direction. As a result, $\mathrm{Pi} 2$ pulsations at BJI in the interval 2100-2300 LT may be affected by at least two factors, the magnetospheric/plasmaspheric cavity (resonance) mode and a surface wave arising at the plasmapause. Thus, Pi2 frequencies may be not only dependent upon the state of the plasmasphere but also their source locations. In other words, the source location may determine the local time dependence of the dominant frequencies of $\mathrm{Pi} 2$ pulsations at low latitudes. In the next subsection, the polarization analysis of two consecutive $\mathrm{Pi} 2$ bursts will be performed to track their source locations.

\subsection{Wave polarization}

In this subsection, the hodograms of the $H$ and $D$ components are used to examine the wave polarization of two consecutive Pi2 bursts. In this study, the azimuthal angle of the major axis of wave polarization is defined as the one between the $+H$ component and the major axis. For the clockwise rotation of the major axis, the azimuthal angle is negative. Otherwise, the azimuthal angle is positive for the counter- 


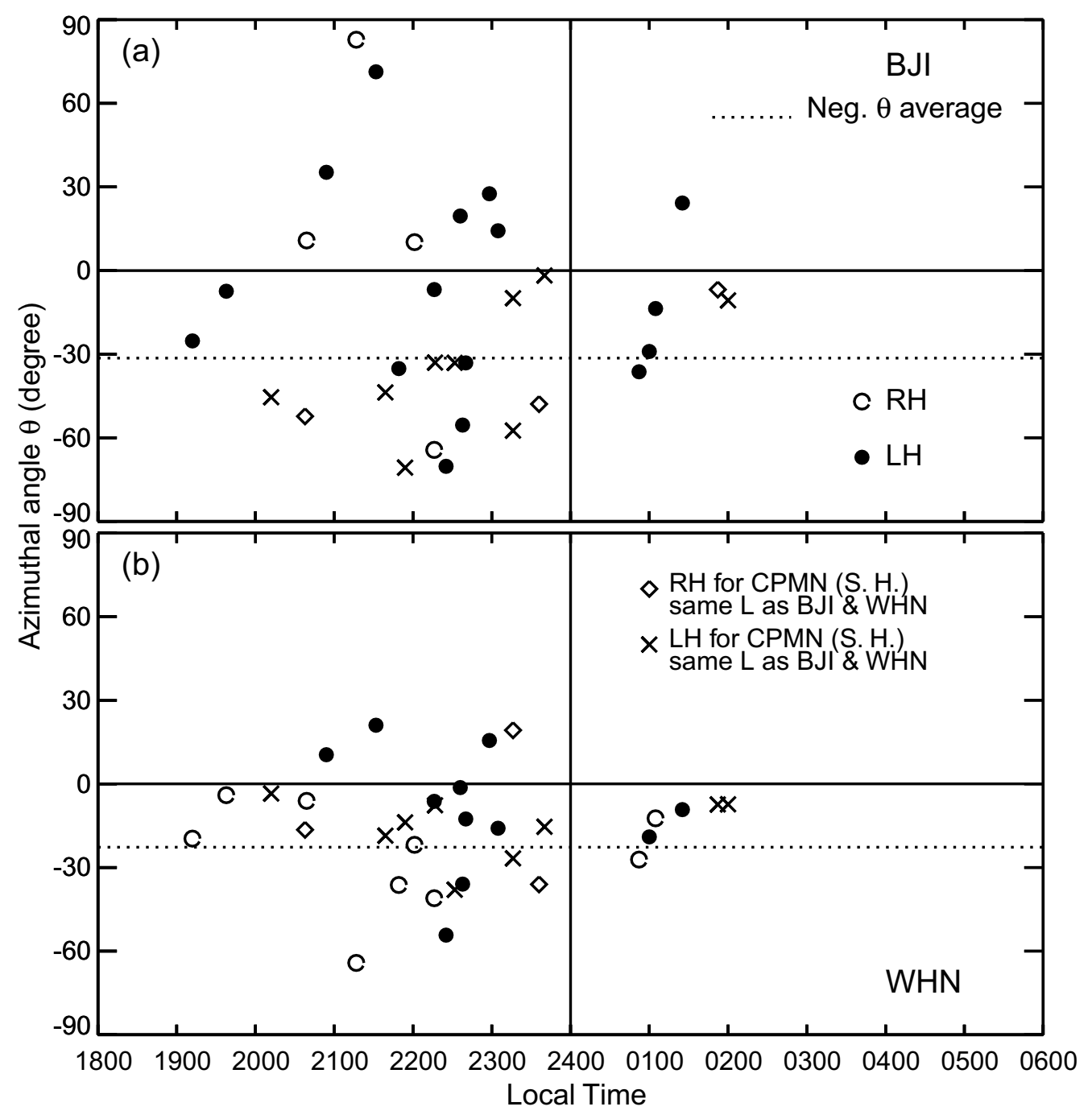

Fig. 5. The azimuthal angle and polarization of two consecutive Pi2 bursts at BJI $(L=1.46)$ and WHN $(L=1.20)$ versus LT. The open circle denotes the right-handed polarization, the solid circle for the left-handed polarization along the geomagnetic field into the north hemisphere, and the dashed line for the average magnitude of the negative azimuthal angle. For the bursts in the south hemisphere, the open diamond denotes the right-handed polarization and the cross for the left-handed polarization.

clockwise rotation of the major axis. By using the BornWolf method (e.g., Rankin and Kurtz, 1970), the azimuthal angle $\theta$ can be calculated with the eigenvector in principal axis analysis.

Figure 5 shows the azimuthal angle and polarization of two consecutive Pi2 bursts at BJI and WHN versus LT, respectively. The pattern of Fig. 5 remains unchanged with inclusion of the data points from the WEP and DAL stations. The open circle denotes the right-handed polarization, the solid circle for the left-handed polarization along the geomagnetic field into the north hemisphere, and the dotted line for the average magnitude of the negative azimuthal angle. In addition, the open diamond denotes the right-handed polarization, the cross for the left-handed polarization along the geomagnetic field into the south hemisphere. Since there are two azimuthal angles (180 apart) that can be used to describe the azimuthal angle and most of azimuthal angles at WHN are negative, the average negative azimuthal angles are consistently used for comparison in this study. Figure 5(a) shows the sense of polarization at BJI becomes alternately right-handed and left-handed between 2100 LT and 2230 LT. In the same format as Fig. 5(a), Figure 5(b) shows the azimuthal angle and polarization of two consecutive Pi2 bursts at WHN versus LT. By comparison of Figs. 5(a) and 5(b), the azimuthal angles at BJI vary drastically in contrast to the averaged negative and approach $90^{\circ}$ compared to those at WHN around 2230 LT. As the geomagnetic activity becomes intensive, the BJI station is closer to the footpoint of the plasmapause than WHN and hence may be dominantly affected by a localized field line oscillation inside the plasmapause. Recently, the correlation study of high- and low-latitude $\mathrm{Pi} 2$ at the $210^{\circ}$ magnetic meridian stations by Yumoto et al. (1994) shows that the latitudinal distribution is different between the $H$ and $D$ components. The $H$-component is symmetric between northern and southern hemispheres and has finite amplitude at the magnetic equator. But the $D$-component is antisymmetric and has a node at the magnetic equator. Later, Itonaga (1995) explained their observation results as a consequence of the field line displacement. This may be the other reason that the azimuth 


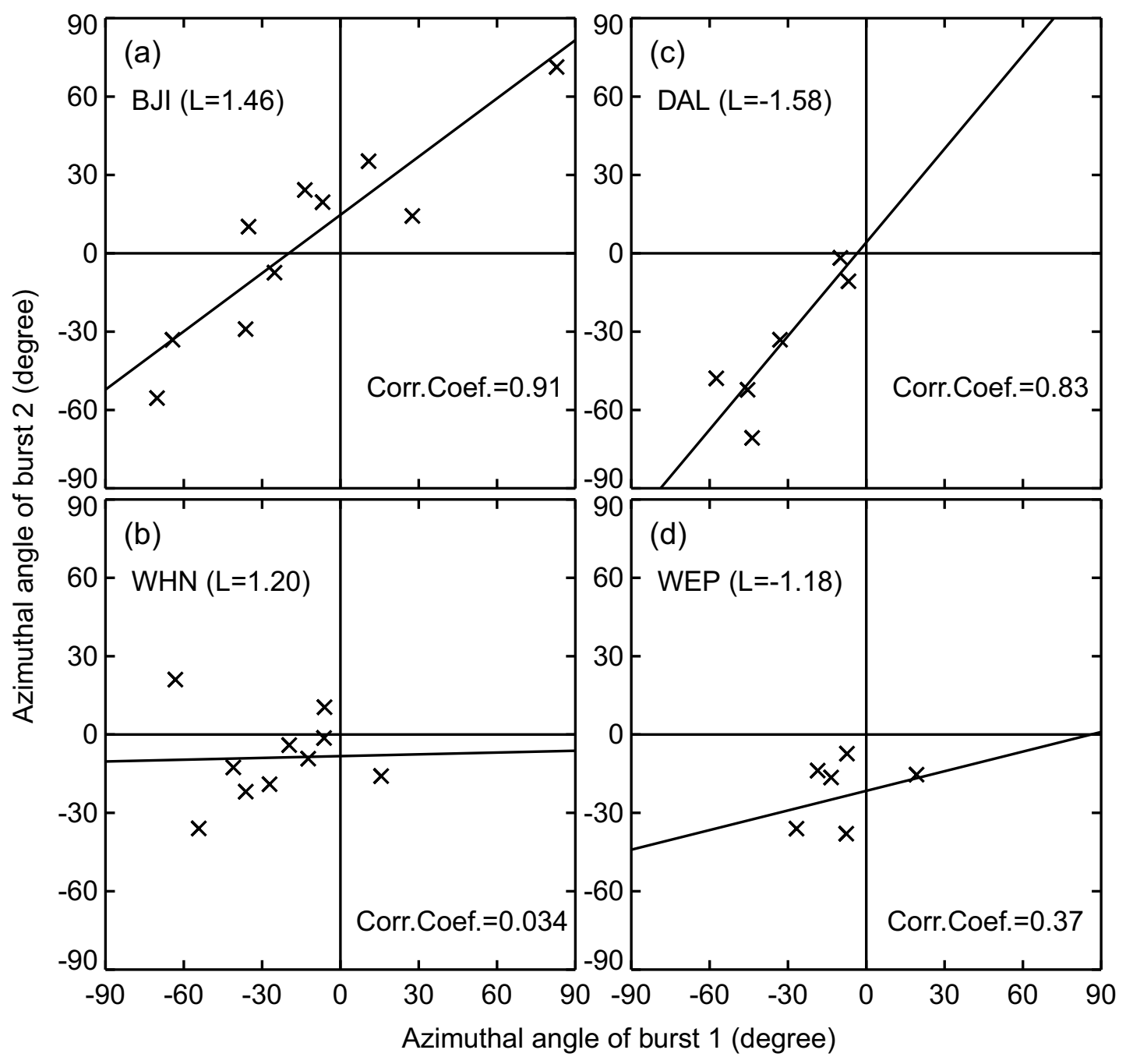

Fig. 6. (a) The azimuthal angle correlation between the first and second Pi2 bursts BJI $(L=1.46)$. (b) In the same format as Fig. 6(a), except for WHN $(L=1.20)$. (c) In the same format as Fig. 6(a), except for DAL $(L=-1.58)$. (d) In the same format as Fig. 6(a), except for WEP $(L=-1.18)$.

at $\mathrm{BJI}$ is closer to $90^{\circ}$ than the WHN station with comparison of Figs. 5(a) and 5(b).

Moreover in Fig. 5, the major axes of Pi2 waves at WHN are mostly aligned in the northeast direction but those at BJI alternate with the northeast and northwest directions. There is also a clear tendency for the local time distribution of the azimuthal angle and polarization at BJI to be more scattered than WHN. This result is similar to those at the mid-latitude station by Villante et al. (1992). From Fig. 5(b), the Pi2 waves at WHN appear right-hand polarized before 2230 LT and left-hand polarized afterwards. Recently, Itonaga and Yumoto (1998) proposed a theory that the sense of rotation of the magnetic perturbation vector in the $H-D$ plane is clockwise (right-hand polarized) and counterclockwise (lefthand polarized) for the eastward and westward propagations in the northern hemisphere. They also noted that the major axis of the $H-D$ plane ellipse is oriented in the northeast and northwest directions west and east of the source longitude in the northern hemisphere. Thus, during substorm onsets, the $\mathrm{Pi} 2$ waves at BJI and WHN may be driven by an impulsive source in the magnetotail in the longitude of 2230 LT and later would propagate westward and eastward as well.

To clarify the difference of local time dependence of the azimuthal angle and polarization between BJI and WHN, the correlation between the first Pi2 burst and the second is studied and shown in Fig. 6. In comparison between Fig. 6(a) and Fig. 6(b), the correlation coefficient of two consecutive $\mathrm{Pi} 2$ bursts at BJI is 0.91 larger than 0.034 at WHN. For further verification, the six same events in the south hemisphere are analyzed in the same way. Similarly, the comparison between Figs. 6(c) and 6(d) shows that the correlation coefficient at DAL is 0.83 larger than 0.37 at WEP. According to Itonaga and Yumoto (1998), the geomagnetic perturbation at middle and low latitudes has mainly two parts: one part is due to the ionospheric current in the polar region and the other part is the mixture of the fast compressional wave and the shear Alfv́en wave excited through wave coupling after substorm onset. If the shear Alfvén wave is dominant, the geomagnetic perturbation will be polarized with a large azimuth and show a horizontal phase propagation. Otherwise, the geomagnetic perturbation will be polarized with a small azimuth while dominated by the fast compressional wave. If the substorm current wedge and/or a localized field line oscillation driven by the shear Alfvén wave occur near the plasmapause, the azimuth of $\mathrm{Pi} 2$ polarizations at higher latitudes should be larger than that at lower latitudes 


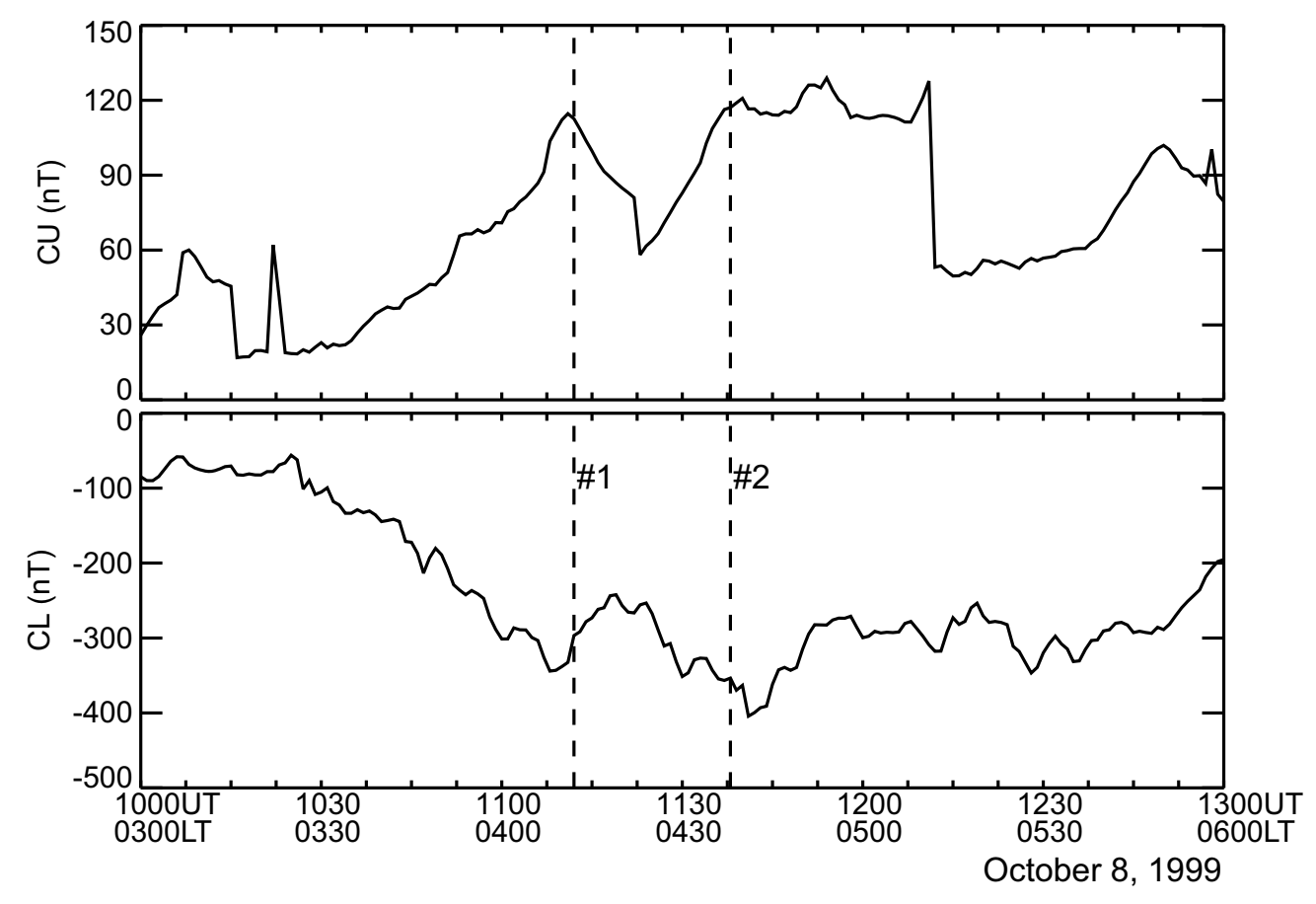

Fig. 7. The $A E$ index at CANOPUS from 1000 UT (0300 LT) to 1300 UT (0600 LT) on October 8, 1999. The dashed line denotes the onset time of two Pi2 bursts at BJI $(L=1.46)$ and WHN $(L=1.20)$. \#1 denotes the first Pi2 burst and \# 2 for the second.

where the fast compressional wave is dominant. As aforementioned, the BJI and DAL stations are closer to the footpoint of the plasmapause than WHN and WEP. By contrast, one may find in Fig. 5 that the azimuth of Pi2 polarizations at BJI and DAL becomes larger than that at WHN and WEP around 2230 LT. Accordingly, two consecutive Pi2 bursts at BJI and DAL may be more affected by the substorm current wedge and/or a localized field line oscillation inside the plasmapause than those at WHN and WEP where fast compressional waves may be predominant instead. This may be the reason why the correlation coefficient between two Pi2 bursts at BJI and DAL is higher than that at WHN and WEP. In the following section, to justify the view, we will undertake the October 8, 1999 event study by comparing the magnetic field data from the stations at CPMN, IGPP/LANL, and geostationary satellites GOES 8 and GOES 10 to those at SMALL.

\section{October 8, 1999}

According to the IMF observation at Wind which was situated at $\sim 47 R_{E}$ in front of the Earth (not shown in this study), the IMF turned southward at $\sim 0834$ UT, sporadically northward and southward before $\sim 1030$ UT, and remained southward until $\sim 1230$ UT for more than 4 hours on October 8,1999 . Therefore a substorm onset might be expected to occur during the time of interest. To test our expectation, the $A E$ index at CANOPUS from $1000 \mathrm{UT}$ (0300 LT) to 1300 UT $(0600$ LT) is plotted in this study. Figure 7 shows that there are two sharp declines in CL right after onsets of two consecutive Pi2 bursts at BJI and WHN. To check if the Pi2 waves propagate globally, the magnetic field data at both the Athabasca (ATH, CGM lat. $62.31^{\circ}$, long. $305.56^{\circ} ; L=4.63$ ) and Air-Force Academy stations (AFA, CGM lat. $48.05^{\circ}$, long. $320.22^{\circ} ; L=2.24$ ) in IGPP/LANL are compared to those at SMALL. The geographic locations of the ATH and AFA stations are shown in Table 2. Figure 8 shows the time derivative of $H$-component with the simulation of a search coil magnetometer with a band pass up to $0.125 \mathrm{~Hz}$. In Fig. 8 , two consecutive Pi2 bursts simultaneously occur at two twin stations in a span of at least $100^{\circ}$ geomagnetic longitude. Figure 8 also shows that two consecutive Pi2 bursts at ATH are out of phase in contrast to other three stations inside the plasmasphere that are in phase with one another.

Furthermore to see if the phase change of the Pi2 waves seen between inside and outside the plasmapause is a global phenomenon, the time derivatives of $H$-component at CPMN same as Fig. 8 are plotted in this study as well. Figure 9 shows that two consecutive Pi2 bursts simultaneously occur from the high- to low- $L$ stations at CPMN. The amplitude of two Pi2 bursts at CPMN also decreases from high- to low-L stations except for KTN, probably out of the footpoint of the impulsive source site in the magnetotail. Between MGD $(L=2.86)$ and CHD $(L=5.58)$, they have roughly out of phase relation like the one reported by Yumoto et al. (1994). This indicates that two consecutive Pi2 bursts have a phase change around ZYK $(L=3.97)$ possibly close to the footpoint of the plasmapause. Figure 9 also shows that the waveforms of two consecutive Pi2 bursts are same for most stations at CPMN except for PTK due to the wrong timing. But for the first burst, one may find that in the beginning the amplitude at ZYK is smaller than that at MGD. Compared to other low-latitude stations at CPMN, the amplitude at MGD is apparently the largest. For the first burst, the amplitude enhancement and phase change around $L=2.86$ are similar to the one studied by Cheng et al. (1998). By comparing ground observations to the simulation results in a box model, Cheng et al. (1998) suggested that the amplitude enhancement and phase change result from the coupling of 


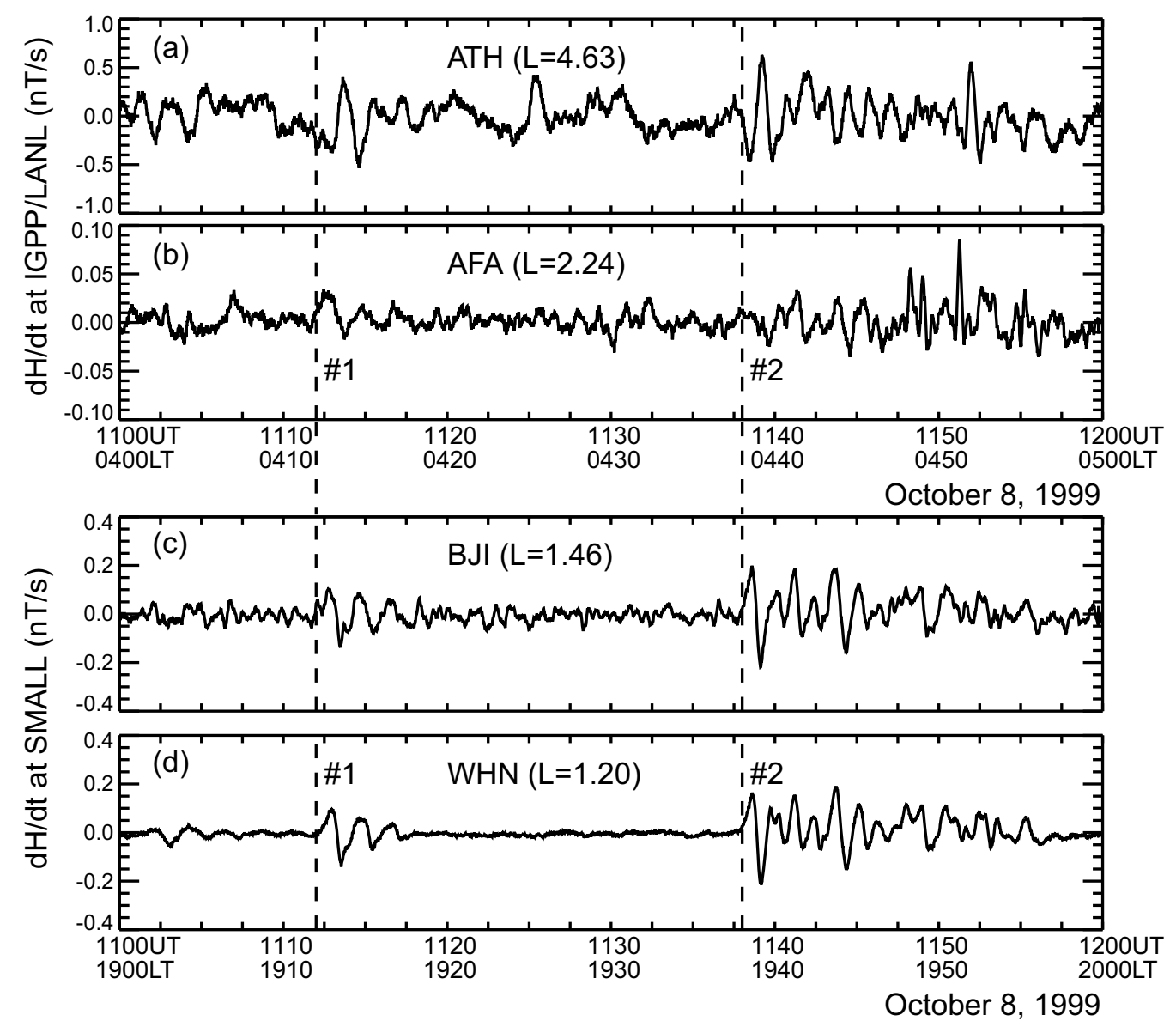

Fig. 8. The time derivatives of $H$-component at ATH $(L=4.63)$, AFA $(L=2.24)$, BJI $(L=1.46)$ and WHN $(L=1.20)$ from $1100 \mathrm{UT}$ to $1200 \mathrm{UT}$ on October 8, 1999. The dashed line denotes the onset time of two Pi2 bursts at BJI and WHN. \#1 denotes the first Pi2 burst and \#2 for the second.

a fast magnetospheric cavity (resonance) mode to field line resonances in the inner magnetosphere. Thus same as Itonaga and Yumoto (1998) pointed out, mid- and low-latitude $\mathrm{Pi} 2 \mathrm{~s}$ may be the mixture of the fast magnetospheric cavity (resonance) mode and the shear Alfvén wave.

The power spectra of two consecutive Pi2 bursts of seven CPMN stations inside and outside the plasmapause are reanalyzed with FFT adopted by Cheng et al. (2000) and shown in Fig. 10. Note that the solid line deontes burst 1 and the dashed line for burst 2. One may find from Fig. 10 that there is a dominant peak at the frequency of $3 / 256 \mathrm{~Hz}$ at all stations for burst 1. But for burst 2, the power spectrum at ZYK ( $L=3.97$ ) close to the plasmapause is not same as those at other stations that have more than two harmonic peaks. Moreover, the power spectrum of burst 2 at CHD $(L=5.58)$ similar to that at MGD $(L=2.86)$ has three harmonic peaks of which the first has the frequnency of $2 / 256 \mathrm{~Hz}$ not seen by the MSR $(L=1.59)$ and KAG ( $L=1.21)$ stations. These confirm once again the findings by Cheng et al. (2000) that low-latitude $\mathrm{Pi} 2$ pulsations on the nightside may be propagating via the plasmaspheric cavity (resonance) mode resulting from fast compressional waves from the magnetotail during substorm onset. The comparison of Fig. 8 to Fig. 9, however, shows that the $\mathrm{Pi} 2$ amplitude at AFA is quite a bit smaller than that at BJI and WHN as well as those at the CPMN stations with $L<3.97$. Note that the AFA station was in the postmidnight sector but the
SMALL and CPMN stations were in the premidnight sector. This also justifies the view from Fig. 5 that the impulsive source in the magnetotail may commence in the longitude of 2230 LT. Owing to the plasma injection at the time of successive substorm onsets, the plasma density at the outer boundary of the inner magnetopshere would be higher than that at the plasmapause. Then the fast compressional wave could be more easily trapped in the inner magnetosphere than in the plasmasphere. Therefore the strength of the plasmaspheric cavity (resonance) mode may decline in contrast to the magnetospheric cavity (resonance) mode away from the impulsive source site. Hence, the substorm current wedge oscillation and the magnetospheric cavity (resonance) mode may be two dominant components for low-latitude Pi2 away from the source longitude during substorm onset.

To find what is exactly the generation mechanism of lowlatitude Pi2, we will compare the ground observation to the magnetic field data at two synchronous orbit satellites GOES 8 and GOES 10. During the time of interest, GOES 8 was moving from $(-1.35,-6.40,1.03) R_{E}$ to $(4.88,-4.35,1.01)$ $R_{E}$ in the GSM coordinate system. At the mean time, GOES 10 was moving from $(-6.27,-2.09,-0.25) R_{E}$ to $(-3.05$, $-5.85,0.55) R_{E}$. In other words, GOES 8 was orbiting from the dawn sector to the morning sector and GOES 10 was going into the dawn sector from the postmidnight sector. The magnetic field at GOES 8 and GOES 10 satellites is defined as: $H p$, perpendicular to the satellite orbital plane (or paral- 


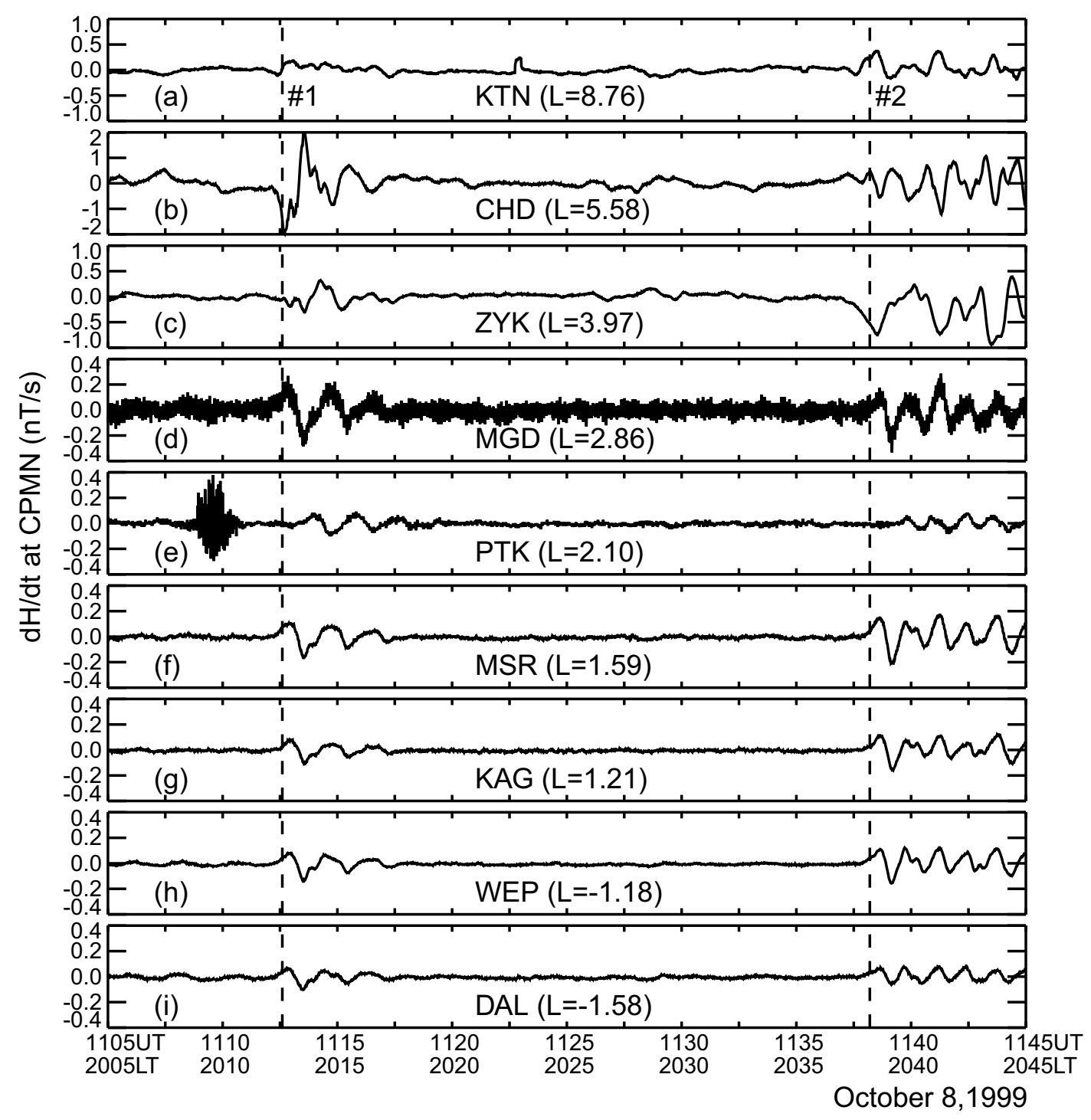

Fig. 9. The time derivatives of $H$-component at CPMN from the high- to low- $L$ stations in the north hemisphere and two stations in the south hemisphere from 1105 UT (2005 LT) to 1145 UT (2045 LT) on October 8, 1999. The dashed line denotes the onset time of two Pi2 bursts at BJI and WHN. \#1 denotes the first Pi2 burst and \#2 for the second.

lel to the Earth spin axis in the case of a zero degree inclination orbit); $\mathrm{He}$, perpendicular to $\mathrm{Hp}$ and directed earthwards; and $H n$, perpendicular to $H p$ and directed eastwards. Figures 11(a) and 11(d) show that at the onsets of two Pi2 bursts the $\mathrm{He}$-component simultaneously begins to decrease at GOES 8 and GOES 10. And Figures 11(c) and 11(f) show that the $\mathrm{Hp}$-component begins to increase as well for two satellites. This signals the dipolization of geomagnetic configuration in the nightside magnetosphere during substorm onset. Figures 11(b) and 11(e) show that the increase in fluctuations in the $\mathrm{Hn}$ direction confirms the onset of activity signaled by the $\mathrm{Pi} 2$ on the ground. Moreover at GOES 8 and GOES 10 , the $H n$-component at onset has positive perturbations and the $\mathrm{He}$-component has negative perturbations. These results are similar to those studied by Cheng et al. (2002b) and confirm the formation of the substorm current wedge after onsets of two Pi2 bursts. Although GOES 8 was at the dawn sector during the time of interest, the effect of the substorm current wedge could continue from the nightside to the dayside hemisphere. This justifies the possibility that a transmission of the electric field from the nightside auroral latitude to the equatorial ionosphere may evoke the occurrence of the dayside Pi2 (Yumoto and the CPMN Group, 2001).

Multipoint observations of the October 8, 1999 event on the ground and in space verify that two consecutive Pi2 bursts at BJI and WHN are the global phenomena. As a result, Pi2 pulsations on the nightside may result from hydromagnetic waves driven by an impulsive source in the magnetotail in the longitude of 2230 LT and later propagate westward and eastward as well. Low-latitude Pi2 near the source site may be made up of at least three components including the substorm current wedge oscillation, the surface wave at the plasmapause or a localized field line oscillation inside the plasmapause, and the magnetospheric/plasmaspheric cavity (resonance) mode. But away from the source longitude, the substorm current wedge oscillation and the magnetospheric cavity (resonance) mode are two main components for lowlatitude $\mathrm{Pi} 2$ on the nightside. 


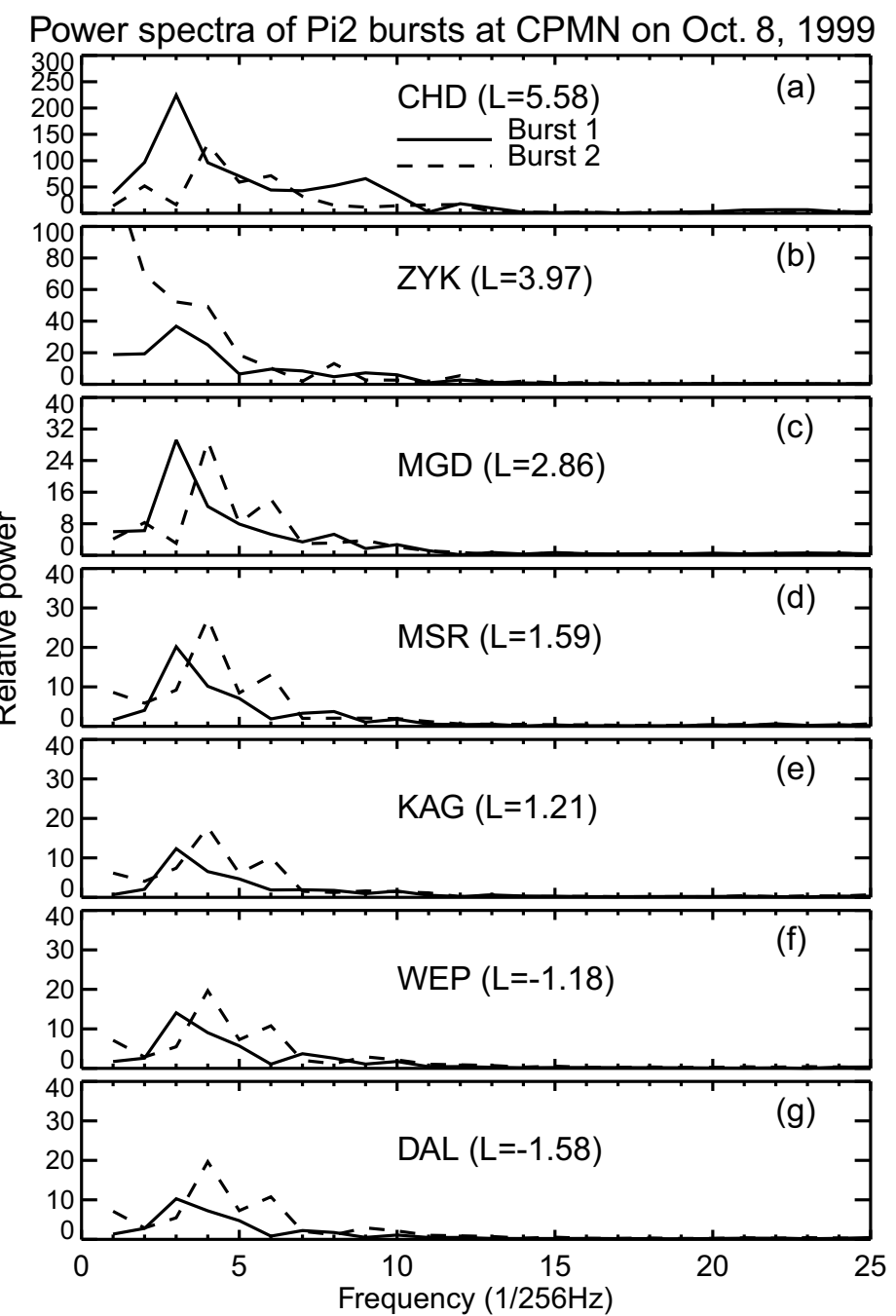

Fig. 10. Power spectra of two consecutive Pi2 bursts of seven CPMN stations inside and outside the plasmapause on October 8, 1999. The solid line denotes the first burst and the dashed line for the second.

\section{Discussion and Summary}

After the substorm expansion onset, magnetic energy released by reconnection in the near Earth neutral line accelerates the plasma flows. These flows are called bursty bulk flows and some move earthward and other tailward. The BBF may move close to the Earth in the longitude of 21002300 LT and brake near the plasmapause due to the balance of magnetic pressure and flow kinetic pressure while the $K p$ index becomes higher. As a result, at least two kinds of hydromagnetic waves are driven impulsively by the BBF. One is the fast compressional wave, propagating from the BBF braking region and bouncing back from the Earth. During the lifetime of the $\mathrm{BBF}$, fast compressional waves are trapped in the inner magnetospheric cavity and result in the cavity resonance (e.g., Cheng et al., 2000; and references therein). In this study, Figure 4 shows the dominant frequencies of two consecutive $\mathrm{Pi} 2$ bursts at WHN are higher than those at BJI. This indicates that the cavity (resonance) mode may play a dominant role in consecutive $\mathrm{Pi} 2$ bursts at WHN. Moreover, the braking BBFs may stimulate the surface wave at the plasmapause with the steep gradient of Alfvén speed. For consistency with the estimated frequency, $\mathrm{Pi} 2$ pulsations at BJI may be the surface wave that is excited at the plasma- pause in the longitude of 2230 LT (see Fig. 5(a) in this study).

The other is the shear Alfvén wave accounting for a localized field line oscillation inside the plasmapause results from the coupling of the fast magnetospheric cavity (resonance) mode to field line resonances as the eigenfrequency is met. In Fig. 9, the amplitude enhancement and phase change of the first Pi2 burst at the station near the footpoint of the plasmapause is similar to the one studied by Cheng et al. (1998) due to the coupling of a fast magnetospheric cavity resonance mode to field line resonances in the inner magnetosphere. Thus, mid- and low-latitude Pi2s may be composed of the shear Alfvén wave and the magnetospheric cavity (resonance) mode.

In Fig. 9, one also can see that the period of the second burst is shorter than the first one. If a fast magnetospheric cavity (resonance) mode is excited by the impulsive source in the magnetotail after substrorm onset, its eigenfrequency should be determined by the size and structure of the resonant cavity. After the first onset, the geomagnetic configuration becomes more dipolarized. The outer boundary of the inner magnetopsheric cavity for the second $\mathrm{Pi} 2$ burst would move more earthward than that for the first one. Therefore, the dominant frequency of the second burst would become 

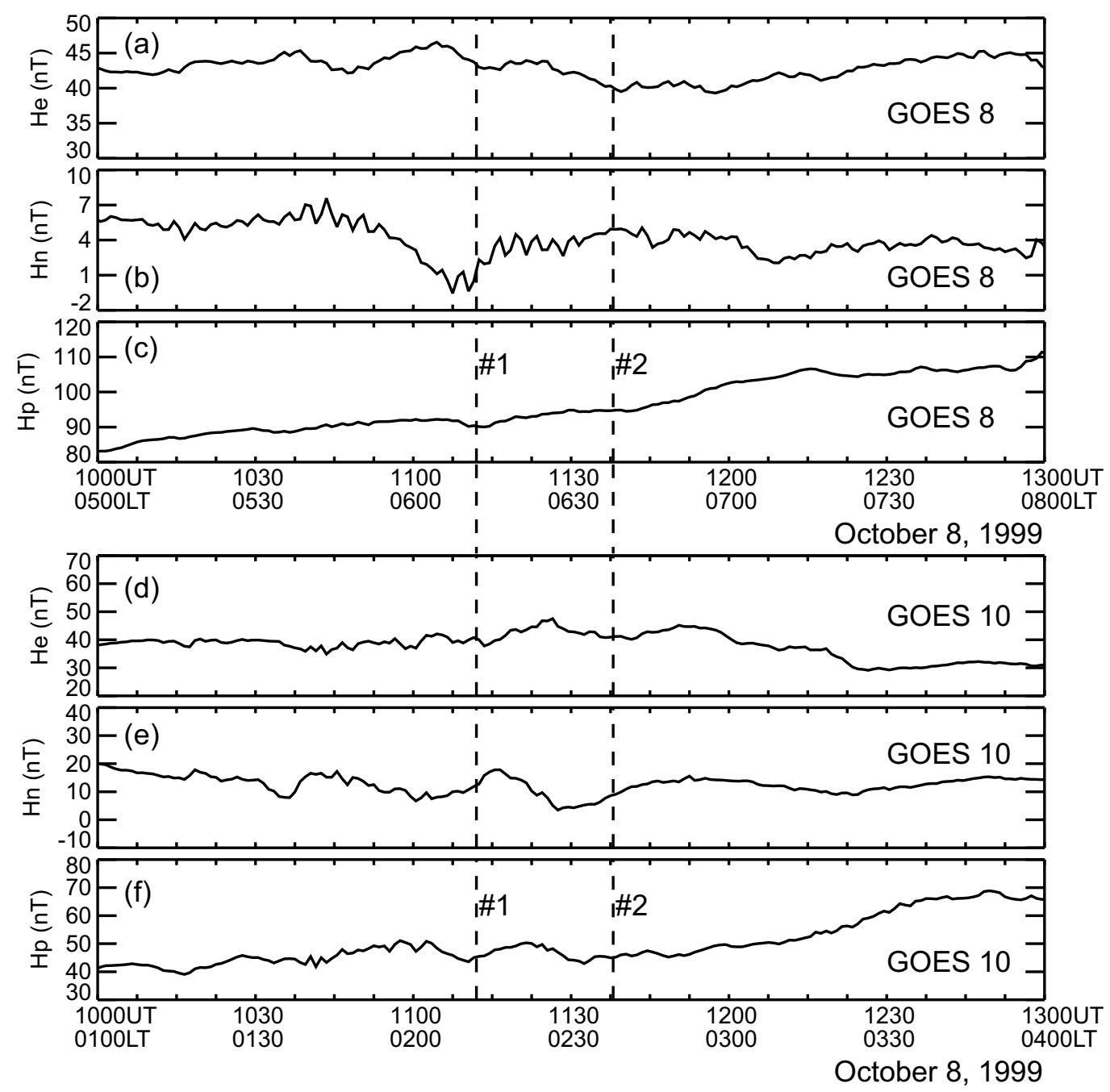

Fig. 11. (a) The $H e$-component of GOES 8 from 1000 UT (0500 LT) to 1300 UT (0800 LT) on October 8 , 1999. During the interval, GOES 8 was moving from $(-1.35,-6.40,1.03) R_{E}$ to $(4.88,-4.35,1.01) R_{E}$ in the GSM coordinates. \#1 and \#2 denote ground Pi 2 bursts, respectively. The dashed line denotes the onset time for two Pi2 bursts at SMALL. (b) Same as Fig. 11(a), except for the $H n$-component. (c) Same as Fig. 11(a), except for the $H$ p-component. (d) In the same format as Fig. 11(a), except for GOES10 and in the interval 0100-0400 LT. In the mean time, GOES 10 was moving from $(-6.27,-2.09,-0.25) R_{E}$ to $(-3.05,-5.85,0.55) R_{E}$ in the GSM coordinates. (e) Same as Fig. 11(d), except for the Hn-component. (f) Same as Fig. 11(d), except for the $H$ p-component.

higher than the first one. If the earthward BBFs move to the flank before approaching the plasmapause, the plasma density at the outer boundary of the inner magnetopshere would be higher than that at the plasmapause. Then the fast compressional wave could be more easily trapped in the inner magnetosphere than in the plasmasphere. If the earthward BBFs spread in the wide longitudinal extent, the strength of the magnetospheric cavity (resonance) mode would be stronger than the plasmaspheric cavity (resonance) mode. This may be the other reason why the amplitude of lowlatitude $\mathrm{Pi} 2 \mathrm{~s}$ tends to decrease from pre-midnight to postmidnight (see Fig. 9 in this study). The similar trend can be found in Fig. 3 of the Nosé et al. (2003) study except from the post-midnight to the morning. Based on the same waveforms and no phase delays between the multipoint ground and satellite observations, however, Nosé et al. (2003) suggested that low-latitude Pi2 pulsations could extend to the morning side and might result from the plasmaspheric cavity (resonance) mode. For this event occurring in the geomagnetic quiet periods, Nosé et al. (2003) inferred that the plasmapause location could expand to large $L$ from the nightside to the morning. With the analysis of wave modes observed by CRRES, Takahashi et al. (2001) suggested that the outer limit of the inner magnetosphere is the plasmapause. When the geomagnetic activity is low, there may be no clear difference between the magnetospheric cavity (resonance) mode and the plasmaspheric cavity (resonance) mode.

According to Shiokawa et al. (1998), the braking of the BBF may be responsible for the field-aligned current and form the substorm current wedge by the closure of the westward traveling surge in the auroral ionosphere. In Figs. 11(d) and 11(e) of this study, magnetic field perturbations at GOES 10 show that the $\mathrm{Hn}$-component has positive perturbations and the $\mathrm{He}$-component has negative perturbations after each $\mathrm{Pi} 2$ onset. These signify the formation of the substorm current wedge after the onsets of two Pi2 bursts. Moreover compared to other high-latitude stations at CPMN, the $H$ component of magnetic field at CHD decreases and reaches the minimum (see Fig. 1 in this study), the $D$-component at 
TIK decreases and that at CHD increases (see Fig. 2 in this study), and the $Z$-component at CHD increases and that at ZYK decreases (see Fig. 3 in this study). These imply that the westward traveling surge may be located between CHD and ZYK and the field-aligned current may go upward over CHD. Hence, the BBF model may be the possible generation scenario for two consecutive Pi2 bursts at SMALL.

Recently by referring to the ground observation in the same meridian near the midnight, Yamaguchi et al. (2002) examined the timing relationship between $\mathrm{Pi} 2 \mathrm{~s}$ at the synchronous orbit and BBFs in the magnetotail. They found that the ground $\mathrm{Pi} 2$ signatures were correlated much better with those at the synchronous orbit than with BBFs. Consequently, they suggested that low-latitude $\mathrm{Pi} 2$ pulsations may not be directly excited by BBFs. In addition to BBFs, the current disruption in the magnetotail is the other impulsive source associated with the geomagnetic reconfiguration after substorm onset. Assuming the impulsive source current as a wave generator in the magnetotail, Fujita et al. (2002) and Fujita and Itonaga (2003) simulated the behavior of hydromagnetic waves in a model magnetosphere with a longituinally non-uniform plasmasphere between $L=3$ and 16.2 after substorm onset. Their simulation results show that the major axis of polarization of the magnetic perturbation like $\mathrm{Pi} 2$ pulsation is radially oriented at midnight and azimuthally oriented at dawn and dusk. They also found that the cavity (resonance) mode in a $\mathrm{Pi} 2$ period could sustain in the plasmapshere and the field line resonance could be excited at the regions with the steep gradient of Alfvén speed as well. Further, the plasmaspheric cavity (resonance) mode could have local time depending spectra like those observed by the ground stations. In addition, the localized field line resonance mode could have the same tendency of local time depending spectra as the plasmaspheric cavity (resonance) mode. Unlike the cavity mode, the surface wave is an evanescent fast mode and its amplitude decreases as it propagates isotropically. Until now, the longitudinal extent of localized Pi2s is not well determined. Fujita and Itonaga (2003) suggested that the surface wave could be a possible generation mechanism for Pi2s localized in a longitudinal direction. This may be the reason why the amplitude of low-latitude Pi2s tends to decrease from pre-midnight to post-midnight (see Fig. 9 in this study). The simulation features by Fujita et al. (2002) and Fujita and Itonaga (2003) are qualitatively consistent with the observational results in this study. Hence, the current disruption in the magnetotail may be the other possible source candidate for two consecutive Pi2 bursts at SMALL.

Until now, there are no concrete conclusions on interpreting the nature of Pi2s at low latitudes from their wave polarizations. However, the orientation of the major axis can still provide some information about their generation and propagation mechanisms. By comparing Fig. 5(a) to Fig. 5(b), one may find that the azimuth versus LT distribution at BJI is more scattered than WHN. The azimuth distribution at $\mathrm{BJI}$ is similar to the one at the mid-latitude station reported by Villante et al. (1992). The azimuth of two consecutive Pi2 bursts versus LT distribution at WHN is also similar to their observation results at the low-latitude station. Villante et al. (1992) suggested that the Pi2 azimuth at the mid-latitude sta- tion might be more affected by the substorm current wedge than that at the low-latitude station. Since the SMALL stations are usually inside the plasmasphere, the Pi2 azimuth at BJI and WHN may be more controlled by a localized field line oscillation inside the plasmapause than the substorm current wedge.

In Fig. 5(b), the Pi2 waves at WHN appear right-hand polarized before 2230 LT and left-hand polarized afterwards. According to Itonaga and Yumoto (1998), hydromagnetic waves may be driven by an impulsive source in the magnetotail which could commence in the longitude of 2230 LT and later propagate eastward with the sense of right-hand polarization in the $H-D$ plane and westward for the left-hand polarization instead. Moreover, Fig. 5(b) is consistent with the observational result reported by Yumoto and the CPMN Group (2001) that Pi2 in the pre-midnight sector occurs earlier than that in the evening sector. But in Fig. 5(a), the major axes of $\mathrm{Pi} 2$ waves at $\mathrm{BJI}$ alternate with the northeast and northwest directions during 2100-2300 LT. In this study, most of Pi2 bursts occurred in the 2100-2300 LT zone where the westward traveling surge may be located and the fieldaligned current may go upward over as well (see Figs. 1, 2, and 3 in this study). Thus we cannot rule out the possibility that the substorm current wedge may result in the apparent azimuth variation of $\mathrm{Pi} 2$ at $\mathrm{BJI}$ in contrast to WHN. In other words, the control of $\mathrm{Pi} 2$ azimuth at WHN by a localized field line oscillation inside the plasmapause is more obvious than that at BJI. As a result, low-latitude Pi2 near the source site may be composed of at least three components including the substorm current wedge oscillation, the surface wave at the plasmapause or a localized field line oscillation inside the plasmapause, and the magnetospheric/plasmaspheric cavity (resonance) mode. But away from the source longitude, the substorm current wedge oscillation and the magnetospheric cavity (resonance) mode may be the main component for low-latitude $\mathrm{Pi} 2$ on the nightside.

Except for the substorm current wedge oscillation, other factors responsible for low-latitude $\mathrm{Pi} 2$ are closely associated with the plasmapause location. The plasmasphere shape changing with time may lead to the local time dependence of the dominant frequency of low-latitude Pi2s. Recent imaging measurements at the spacecraft (e.g., Burch et al., 2001) showed that the plasmapshere rarely had a smooth steady shape and even a plasma tail in the dusk sector. The asymmetric plasmapshere may not vary uniformly so that the difference of the plasmapause distance might be mainly a temporal effect. Therefore the temporal variation of the plasmapause location may result in the slight difference of the dominant frequency of Pi2s between the low-latitude sites in the different longitude. To discern which factor is most dominant, the latitudinal and longitudinal dependences of the dominant frequency and polarization characteristics of lowlatitude Pi2s need further examination on both dense ground measurements and coordinated satellite observations from outside and inside the plasmapause down to the low latitudes in the future study.

In summary, with reference to $H$-component magnetic bays at CPMN, 10 events of consecutive $\mathrm{Pi} 2$ pulsations are simultaneously observed by BJI and WHN in association with substorm onsets. Multipoint observations of the Oc- 
tober 8, 1999 event on the ground and in space verify that two consecutive Pi2 bursts at BJI and WHN are the global phenomena. Their occurrences are mostly in the 2100-2300 LT sector in which the dominant frequencies at WHN are higher than the mean frequency but those at BJI are lower and close to the frequency of the surface wave at the plasmapause. The azimuthal angles of $\mathrm{Pi} 2$ waves are less correlated to each other at WHN than at BJI. The LT dependence of azimuth and polarization of consecutive $\mathrm{Pi} 2$ pulsations at BJI and WHN can be interpreted with the theoretical model by Itonaga and Yumoto (1998). GOES 8 and GOES 10 confirm the formation of substorm current wedge after the onsets of two Pi2 bursts. During substorm onsets, Pi2 pulsations at low latitudes may result from hydromagnetic waves driven by an impulsive source in the magnetotail which could commence in the longitude of $2230 \mathrm{LT}$ and later would propagate westward and eastward as well. For low-latitude Pi2 near the source site, the surface wave at the plasmapause, a localized field line oscillation inside the plasmapause, and the magnetospheric/plasmaspheric cavity (resonance) mode are three important factors in affecting their waveforms. But for lowlatitude Pi2 away from the source, the magnetospheric cavity (resonance) mode may be the main factor instead.

Acknowledgments. We thank all members of the CPMN project for their ceaseless support. We acknowledge T. Uozumi for handling the CPMN data archives. The magnetometer data at the ATH and AFA stations were obtained with assistance from M. Connors and F. K. Chun. The $K p$ index data were provided by National Geophysical Data Center, NOAA, Boulder, Colorado at the website (http://www.ngdc.noaa.gov/stp). The $A E$ index data at CANOPUS were provided by G. Rostoker at University of Alberta in Canada and CDAWeb. The magnetic field data of GOES 8 and GOES 10 were provided by H. Singer at NOAA on the CDAWeb. This work was supported by National Science Council of R. O. C. on Taiwan under the grant NSC 92-2111-M-150-001.

\section{References}

Bauer, T. M., W. Baumjohann, and R. A. Treumann, Neutral sheet oscillations at substorm onset, J. Geophys. Res., 100, 23,737-23,742, 1995.

Baumjohann, W. and K. H. Glassmeier, The transient response mechanism and $\mathrm{Pi} 2$ pulsations at substorm onset-Review and outlook, Planet. Space Sci., 32, 1361-1370, 1984.

Burch et al., Global dynamics of the plasmasphere and ring current during magnetic storms, Geophys. Res. Lett., 28, 1159-1162, 2001.

Cheng, C.-C., J.-K. Chao, and T.-S. Hsu, Evidence of the coupling of a fast magnetospheric cavity mode to field line resonances, Earth Planets Space, 50, 683-697, 1998.

Cheng, C.-C., J.-K. Chao, and K. Yumoto, Spectral power of low-latitude $\mathrm{Pi} 2$ pulsations at $210^{\circ}$ magnetic meridian stations and plasmaspheric cavity resonances, Earth Planets Space, 52, 615-627, 2000.

Cheng, C.-C., C. T. Russell, Y. F. Gao, and P. J. Chi, On consecutive bursts of low-latitude Pi2 pulsations, J. Atoms. Sol. Terr. Phys., 64, 1809-1821, 2002a.

Cheng, C.-C., C. T. Russell, M. Connors, and P. J. Chi, Relationship between multiple substorm onsets and the IMF: A case study, J. Geophys. Res., 107(A10), 1289, doi:10.1029/2001JA007553, 2002 b.

Fujita, S. and M. Itonaga, A plasmaspheric cavity resonance in a longitudinally non-uniform plasmasphere, Earth Planets Space, 55, 219-222, 2003.

Fujita, S., H. Nakata, M. Itonaga, A. Yoshikawa, and T. Mizuta, A numerical simulation of the $\mathrm{Pi} 2$ pulsations associated with the substorm current wedge, J. Geophys. Res., 107(A3), 1034, doi:10.1029/2001JA900137, 2002.

Gao, Y. F., P. J. Chi, G. Le, C. T. Russell, D. M. Yang, X. Zhou, S. F. Yang, V. Angelopoulos, and F. K. Chun, Sino-magnetic array at low latitudes
(SMALL) including initial results from the sister sites in the united states, Adv. Space Res., 25, 1343-1351, 2000.

Itonaga, M., Spatial structure and polarization of geomagnetic pulsations observed on the ground, J. Geomag. Geoelectr., 47, 1023-1027, 1995.

Itonaga, M. and K. Yomoto, ULF waves and the ground magnetic field, $J$. Geophys. Res., 103, 9285-9291, 1998.

Kepko, L. and M. G. Kivelson, Generation of Pi2 pulsations by bursty bulk flows, J. Geophys. Res., 104, 25021-25034, 1999.

Kosaka, K., T. Iyemori, M. Nosé, M. Bitterly, and J. Bitterly, Local time dependence of the dominant frequency of Pi2 pulsations at mid- and lowlatitudes, Earth Planets Space, 54, 771-781, 2002.

Lanzerotti, L. J. and L. V. Medford, Local night, impulsive (Pi2-type) hydromagnetic wave polarization at low latitudes, Planet. Space Sci., 32, 135-142, 1984.

Lee, D. H. and K. Kim, Compressional MHD waves in the magnetosphere: A new approach, J. Geophys. Res., 104, 12,379-12,385, 1999.

Lester, M., H. J. Singer, D. P. Smits, and W. J. Hughes, Pi2 pulsations and the substorm current wedge: Low-latitude polarization, J. Geophys. Res., 94, 17133-17141, 1989.

Li, Y., B. J. Fraser, F. W. Menk, D. J. Webster, and K. Yumoto, Properties and sources of low and very low latitude $\mathrm{Pi} 2$ pulsations, J. Geophys. Res., 103, 2343-2358, 1998.

Lin, C. A., L. C. Lee, and Y. J. Sun, Observations of Pi2 pulsations at a very low latitude $(\mathrm{L}=1.06)$ station and magnetospheric cavity resonances, $J$. Geophys. Res., 96, 21,105-21,114, 1991.

Mishin, V. M., T. Saifudinova, A. Bazarzhapov, C. T. Russell, W. Baumjohann, B. Nakamura, and M. Kubyshkina, Two distinct substorm onsets, J. Geophys. Res., 106, 13,105-13,118, 2001.

Nosé, M, Automated detection of Pi2 pulsations using wavelet analysis: 2. An application for dayside Pi2 pulsations study, Earth Planets Space, 51, 23-32, 1999.

Nosé et al., Multipoint observations of a Pi2 pulsation on morningside: The 20 September 1995 event, J. Geophys. Res., 108(A5), 1219, doi:10.1029/2002JA009747, 2003.

Olson, J. V., Pi2 pulsations and substorm onsets: A review, J. Geophys. Res., 104, 17,499-17,520, 1999.

Rankin, D. and R. Kurtz, Statistic study of micropulsation polarizations, $J$. Geophys. Res., 75, 5444-5458, 1970.

Russell, C. T., Interactive analysis of magnetic field data, Adv. Space Res., 2(7), 173-176, 1983.

Russell, C. T., How northward turnings of the IMF can lead to substorm expansion onsets, Geophys. Res. Lett., 27, 3257-3259, 2000.

Shiokawa, K., W. Baumjohann, G. Haerendel, G. Paschmann, J. F. Fennell, E. Friis-Christensen, H. Luhr, G. D. Reeves, C. T. Russell, P. R. Sutciffe, and K. Takahashi, High-speed ion flow, substorm current wedge, and multiple Pi2 pulsations, J. Geophys. Res., 103, 4491-4507, 1998.

Takahashi, K., S. I. Ohtani, and B. J. Anderson, Statistical analysis of Pi2 pulsations observed by the AMPTE CCE spacecraft in the inner magnetosphere, J. Geophys. Res., 100, 21,929-21,941, 1995.

Takahashi, K., S. I. Ohtani, W. J. Hughes, and B. J. Anderson, CRRES observation of $\mathrm{Pi} 2$ pulsations: Wave mode inside and outside the plasmasphere, J. Geophys. Res., 106, 15,567-15,581, 2001.

Villante, U., M. Vellante, M. De Biase, L. J. Lanzerotti, and L. V. Medford, $\mathrm{Pi} 2$ pulsations at separate site $\left(\Delta \Phi \simeq 90^{\circ}\right)$ : a comparison between midand low-latitude observations, Ann. Geophys., 10, 472-482, 1992.

Yamaguchi et al., The timing relationship between bursty bulk flows and $\mathrm{Pi} 2 \mathrm{~s}$ at the geosynchronous orbit, Geophys. Res. Lett., 29, doi:10.1029/2001GL013783, 2002.

Yumoto, K., Generation and propagation mechanisms of low-latitude magnetic pulsations-A review, J. Geophys., 60, 79-105, 1986.

Yumoto, K., H. Osaki, K. Fukao, K. Shiokawa, Y. Tanaka, S. I. Soloeyev, G. Krymskij, E. F. Vershinin, V. F. Osinin, and $210^{\circ} \mathrm{MM}$ Magnetic Observation Group, Correlation of high- and low-latitude Pi2 magnetic pulsations observed at $210^{\circ}$ magnetic meridian chain stations, J. Geomag. Geoelectr., 46, 925-935, 1994.

Yumoto, K. and the CPMN Group, Chararcteristics of Pi2 magnetic pulsations observed at the CPMN stations: A review of the STEP results, Earth Planets Space, 53, 981-992, 2001.

C.-C. Cheng (e-mail: cccheng@nhust.edu.tw), C. T. Russell, K. Yumoto, Y. F. Gao, and P. J. Chi 